\title{
Sentence interference in the Stroop task
}

\author{
ANGELA G. BREGA and ALICE F. HEALY \\ University of Colorado, Boulder, Colorado
}

\begin{abstract}
In two experiments on Stroop interference, we examined whether sentences can be processed without the intention of the reader. Participants named the ink colors in which words in sentences were printed, and the ink colors in which the same words, randomly arranged, were printed. In Experiment 1, sentences yielded longer response times (RTs) and more errors than did nonsentences, but only when they included words that were highly relevant to the color-naming task (i.e., color and color-related words). In Experiment 2, sentences yielded more errors than did nonsentences, and sentences in which the color words matched the set of ink colors yielded longer RTs than did nonsentences. The results indicate that sentence processing can be obligatory when the component words are highly relevant to the task.
\end{abstract}

In 1935, J. R. Stroop reported a series of experiments that precipitated an explosion of research into what has since been called the Stroop effect. Stroop found that participants took significantly longer to name the ink colors in which incongruent color words were printed than to name the ink colors in which color squares were printed (Experiment 2). Words caused interference in the colornaming task.

According to some explanations of the Stroop effect, individuals are so experienced at word reading that they do it without intention (e.g., Posner \& Snyder, 1975). More recent accounts suggest that the interference evident in the Stroop task is due to vast differences in the relative strengths of processing associated with word reading and color naming (Cohen, Dunbar, \& McClelland, 1990). Processing strength results directly from practice and determines the level of attention that a process requires, the speed with which it can occur, and the interference effects that will emerge in dual-task situations. Word reading is a task practiced frequently; it occurs quickly and requires little attention. Color naming, on the other hand, is a task practiced less often; it therefore requires more time and conscious attention than does word reading.

Because the word-reading and color-naming processes both rely on a common resource (i.e., a verbal response at the output stage), the Stroop task promotes response

Earlier versions of this report were presented at the meeting of the Rocky Mountain Psychological Association in Boulder on April 21, 1995, and at the meeting of the Society of Experimental Psychologists in Laguna Beach, CA on March 28, 1998. This research was supported in part by Army Research Institute Contracts MDA903-93-K-0010 and DASW01-96-K-0010 and by Army Research Office Grant DAAG5598-1-0214 to the University of Colorado. We are indebted to Lyle Bourne, Deborah Clawson, William Marmie, Kathryn Bock, Tram Neill, Padraig O'Seaghdha, Morton Ann Gernsbacher, and anonymous reviewers for their helpful comments on earlier versions of this manuscript, and to James Parker for assistance in the conduct of the experiments. Correspondence concerning this research should be addressed to A. F. Healy, Department of Psychology, University of Colorado, Campus Box 345, Boulder, CO 80309-0345 (e-mail: ahealy@psych. colorado.edu). competition between the two tasks (Cohen et al., 1990). The greater strength of processing associated with word reading gives this task an advantage in the Stroop paradigm, in which it is pitted against a weaker process, color naming. The present study was designed to determine whether such obligatory processing can occur at the level of sentences as well as at the level of individual words.

A word's color relevance determines the level of interference that it causes in the Stroop task. Words that match the set of ink colors used in a stimulus set cause more interference than do words that represent colors not used in printing the stimuli (Klein, 1964). In addition, color words cause more interference than do color-related words (e.g., blood, grass), which, in turn, cause more interference than do noncolor words (e.g., hat, boy) (Dalrymple-Alford, 1972; Klein, 1964). Even noncolor words, however, are associated with significantly longer color-naming response times than are nonsense syllables or nonwords (Bakan \& Alperson, 1967; Klein, 1964).

The heightened interference caused by color-relevant words in the Stroop task may result from spreading activation (Posner \& Snyder, 1975). In studies of the Stroop effect, the color-naming task itself primes color words, facilitating their processing relative to words that are not primed. The more color related a word is, the greater the degree of priming, and thus facilitation, that it receives (MacLeod, 1991). The color relevance of a word, per se, is not the critical feature that determines its ability to produce interference in the Stroop task. After all, in many variations of the Stroop paradigm, color words and color naming are not involved. For example, words representing directional references (e.g., up, left) cause interference in Stroop paradigms in which the task is to name the location in which words occur within stimulus squares (Fox, Shor, \& Steinman, 1971). Whatever the task may be, interference is determined by the word's relevance to the experimental task itself

The present study was designed to determine whether sentence processing can occur without the intention of 
the reader. It was hypothesized that words arranged in meaningful sentences would cause more Stroop interference than would the same words arranged randomly. Sentences were expected to enhance interference in the Stroop task by facilitating the processing of the component words. An individual word may be processed more efficiently when it occurs in a larger context. Text is processed on several linguistic levels (i.e., feature, letter, letter cluster, lexical, syntactic, and semantic) (e.g., Rumelhart, 1977). According to constraint-based models, the processing of these different levels of text occurs in parallel and interacts to produce text comprehension (see, e.g., Tanenhaus $\&$ Trueswell, 1995, for a review of these models and a comparison of them with alternative models).

Words arranged in meaningful sentences and the same words arranged randomly should receive identical processing with regard to the feature, letter, letter cluster, and lexical levels of text processing. Word processing should be enhanced, however, when words occur in sentences, because the reader may engage in syntactic and more exhaustive semantic processing as well as lower order processing. Indeed, the results of syntactic priming studies indicate that response times are faster when target words are preceded by syntactically appropriate primes (e.g., Samar \& Berent, 1986; Sereno, 1991). Likewise, lexical decisions are made more quickly when target words are preceded by semantically related primes (e.g., Sereno, 1991). Further, word recognition is enhanced when words occur in the context of meaningful phrases (e.g., O'Seaghdha, 1989) or sentences (e.g., Hess, Foss, \& Carroll, 1995; Masson, 1986; Simpson, Peterson, Casteel, \& Burgess, 1989).

These studies of syntactic and semantic priming demonstrate that multiword contexts enhance the processing of individual words. All of these studies demonstrate such facilitation in tasks in which experimental participants are instructed to read the stimuli. Because participants' performance on these word recognition and lexical decision tasks is facilitated by their reading the sentence or phrase contexts, the participants have an additional incentive to read the stimuli.

The Stroop task provides a very different test of sentence processing. In this task, participants are instructed not to read the stimuli to which they are exposed, but just to name the ink colors in which those stimuli are printed. Further, attending to the incongruently printed words in the Stroop task inhibits, rather than facilitates, the participants' ability to complete the task successfully. Thus, unlike in semantic and syntactic priming studies, participants in the Stroop task are motivated not to read the words. Using the Stroop task, we can examine sentence processing under conditions in which the reader does not intend to read the sentences. The Stroop task should provide a test of obligatory sentence processing just as it has provided a test of obligatory word reading.

Sentences should be associated with greater interference in the Stroop task than should nonsentences, be- cause activation of the component words is enhanced by syntactic and semantic processing at the sentence level. Sentences enhance the processing of individual words, thus contributing to the ability of those words to interfere with the color-naming task. This enhanced processing of the component words should be especially detrimental to Stroop performance when the sentences contain highly task-relevant words. The heightened activation of taskrelevant words, such as color words, produced by sentence processing should increase the likelihood that these words will compete with the ink color names at the response stage. Therefore, it was hypothesized that words arranged in meaningful sentences would cause more interference than would the same words arranged randomly. In addition, because of the priming received by task-relevant words as a result of spreading activation, it was expected that sentences that contained such words would cause more interference than would sentences composed only of irrelevant (i.e., noncolor) words.

\section{EXPERIMENT 1}

\section{Method}

Participants. Eighty undergraduate students at the University of Colorado participated in partial fulfillment of a course requirement. All participants had normal color vision.

Design, Materials, and Apparatus. The experiment involved a 2 (task relevant and task irrelevant) $\times 2$ (sentence and nonsentence) +1 (control) design. Task relevance was manipulated by generating stimuli that included words highly relevant to the color-naming task (i.e., color words and color-relevant words), and also stimuli that included only words irrelevant to the color-naming task (i.e., noncolor words). The experimental design included five within-subjects conditions: (1) sentences that included color and color-related words (task-relevant sentence condition), (2) sentences composed only of noncolor words (task-irrelevant sentence condition), (3) nonsentences that included color and color-related words (task-relevant nonsentence condition), (4) nonsentences that included only noncolor words (taskirrelevant nonsentence condition), and (5) control stimuli composed of sets of colored bullets (e.g., $\cdots \cdot \cdot \bullet)$.

To control for the effects of sentence structure across the taskrelevant and task-irrelevant conditions, five stimulus sets were generated. Each set included five stimuli, with each stimulus corresponding to one of the five conditions. In each set, the task-relevant and task-irrelevant sentences were identical, with the exception of the color words and color-related words. All noncolor words used in the task-relevant sentences were repeated in the same positions in the task-irrelevant sentences. The syntactic categories of the words in the task-relevant and task-irrelevant sentences were identical. For example, if the first word in a task-relevant sentence was a noun, the first word in the corresponding task-irrelevant sentence was also a noun.

Within each stimulus set, the average frequency of the words in the task-relevant sentence was matched to the average frequency of the words in the task-irrelevant sentence. Word frequency was matched sentence by sentence, not word by word. The average word frequency per million words of text (Kučera \& Francis, 1967) for all words in the task-relevant sentences (color, color related, and noncolor) was 12,317 ; it was 12,318 for the words in the task-irrelevant sentences,

All of the sentences contained nine words. On the average, the task-relevant sentences included $5.6(62 \%)$ color and color-related 
words, with a range from $5(56 \%)$ to $7(78 \%)$. The number of color words used in the task-relevant sentences was nearly equal to the number of color-related words used, with averages of $2.6(29 \%)$ and $3(33 \%)$, respectively.

To control for the effects of word meaning and word frequency across the sentence and nonsentence conditions, the same words were used in both conditions with only the order varying across conditions. In scrambling the order of the words composing the sentence stimuli, it might have been advantageous to keep the positions of the color and color-related words constant across sentence and nonsentence conditions. However, because so many of the words in the task-relevant condition were color and color-related, it was not possible to keep the positions of those words constant. Doing so would have ensured that the "nonsentences" were nearly intact sentences. Therefore, each nonsentence was created by randomizing the order of the words in the corresponding sentence. Appendix A contains all sentence stimuli from each of the five stimulus sets, as well as information regarding the word frequency of the sentences and the number of color and color-related words used.

Each control stimulus included nine groupings of bullets. Bullets were chosen, rather than Xs or asterisks (e.g., Dalrymple-Alford, 1972; Klein, 1964), because they are not readily namable and therefore should cause less interference than more familiar characters or letters (Shadler \& Thissen, 1981). The groupings of bullets varied in length, ranging from 3 to 9 characters, with a mean number of bullets per group of 4.86 . The total number of bullets in the control stimuli ranged from 36 to 48 , with a mean of 43.80 . The five control stimuli were assigned to stimulus sets according to the total number of characters used. The control stimulus with the greatest number of individual bullets was assigned to the stimulus set with the largest number of characters, averaged across the task-relevant and task-irrelevant conditions.

The five stimulus sets, each including five stimuli corresponding to the five experimental conditions, resulted in 25 total stimuli. Each sentence and nonsentence was printed in all capital letters horizontally on an individual $8.5 \times 11$ in. $(21.6 \times 27.9 \mathrm{~cm})$ card. Three ink colors (red, blue, and green) were used to print the stimuli. Four rules were used to determine the ink color in which a given word was printed. First, each of the three colors was used three times in each stimulus. Second, no color was used twice in succession. Third, ink color and word combinations were always incongruent. For example, the word blue, or blue-related words such as sky, was never printed in blue ink. The final rule of ink color assignment was designed to avoid the distractor-suppression (i.e., negative priming) effect. Interference in the Stroop task is enhanced if the to-be-ignored word on trial $n$ matches the to-be-named ink color on trial $n+1$. Suppressing the word red on trial $n$ makes it more difficult to name the color red correctly on the following trial (Dalrymple-Alford \& Budayr, 1966; Neill, 1977). To avoid such interference, the ink color of a given word in a stimulus never matched the color meaning of the preceding word. For example, red ink was never used for the stimulus word immediately following the word red or following a red-related word (e.g., sunset). Even if it is possible for distractorsuppression interference to extend beyond one word (see, e.g., DeSchepper \& Treisman, 1996; Neill \& Westberry, 1987), it was impossible in generating the stimuli to require that the three ink colors always be more than one step behind matching color and color-related words.

Each stimulus in a stimulus set was printed in a unique sequence of ink colors. It was not possible to retain the same sequential order of ink colors across the conditions. In the task-relevant nonsentence condition, it was impossible to randomly reorder the words, meet each of the criteria used for assigning ink colors, and retain the same ink color sequence used in the sentence version. Further, retaining the same sequence of ink colors across the stimuli within a set was not advisable, because repeated exposure to given color sequences might enhance the participants' ability to name the ink colors of stimuli presented later in the Stroop task.
The 25 stimuli were ordered according to a Greco-Latin square design. Each participant was exposed to each of these 25 stimuli. In all, there were five orders in which the stimuli were presented. Sixteen participants were tested with each of these orders. Within each ordering, the stimuli were arranged into five blocks of five stimuli each. A stimulus from each stimulus set was represented in each block, with the stimulus sets always presented in the same order. Each one of the five conditions was represented in each block as well. Within each ordering, each stimulus set was assigned once to each condition across blocks. In each block, each stimulus set was assigned once to each condition across the five orderings.

Procedure. The participants received written as well as oral instructions. The experimenter explained that the participants would see 25 cards, each containing nine items printed in the colors red, blue, or green. The participants were told that these nine items would be groupings of bullets, words arranged randomly, or words arranged in sentences. The participants then received the following instructions:

Whether you are looking at words arranged randomly, sentences, or bullets, your task is to name the ink colors in which each of the nine items on the card are printed. You should ignore the words and just focus on naming the ink colors of the nine items. Speed and accuracy are both important in this task, so please try to name the ink colors as quickly as you can without making any mistakes.

Participants were shown samples of the bullets and Xs printed in the three ink colors. To ensure that the participants had the color vision required to participate in the study, they were asked to name the ink colors in which these sample items were printed. No participants were excluded on the basis of color blindness.

The experimenter recorded errors and, with a stopwatch, the amount of time required for each participant to complete each stimulus card. The stopwatch was started when participants first viewed each card and stopped when they began to utter the name of the ink color in which the final word in the stimulus was printed. An error was scored by the experimenter, who had a listing of the correct color responses, whenever a participant said an inappropriate color name for a given word. Participants' error scores were calculated as the proportion of stimulus cards in a given condition on which at least one error occurred. When instructed to do so, the participants flipped each card over to reveal the next card. Because the participants controlled the cards and the experimenter could not see the stimuli on the cards, the experimenter was unaware of the condition to which participants were responding at any given time.

\section{Results}

Stimulus items on which a participant made one or more errors were removed from the analysis of response times for that participant. Out of the total of 2,000 items, 177 were removed because participants incorrectly named the ink colors. Thirteen items were eliminated because of experimenter errors, 3 were dropped because of participant errors in following the directions, and 30 were dropped because they fell more than three standard deviations away from the mean for each of the 25 stimuli. To maintain control over word frequency and word meaning, in all cases in which an individual response time was dropped, the entire stimulus set to which that item belonged was dropped from the response time analyses for that participant.

The data were analyzed using the subject as the unit of analysis. Formal item analyses were not deemed appropriate because the stimuli were not randomly selected (Wike \& Church, 1976). With regard to the sentence effect, however, informal comparisons focusing on the individual stimulus items will be presented throughout. Ap- 
pendix A contains the mean response times and error frequencies by condition for each stimulus set.

Response time. Because response times are often characterized by nonnormal distributions, they were analyzed using both the raw data and data transformed to the natural log with base $e$ (Judd \& McClelland, 1989). The results were identical for these separate analyses. Therefore, only the analyses for the untransformed response times are presented here. The means and standard errors of the mean of the response time and error rate data are presented in Table 1. Individual contrasts indicated that each of the four experimental conditions resulted in significantly longer response times than did the bullet control condition [task-relevant sentence, $F(1,79)=$ $314.74, M S_{\mathrm{e}}=.22, R^{2}=.80, p<.001$; task-relevant nonsentence, $F(1,79)=172.10, M S_{\mathrm{e}}=.26, R^{2}=.69, p<$ .001 ; task-irrelevant sentence, $F(1,79)=88.37, M S_{\mathrm{e}}=$ $.09, R^{2}=.53, p<.001 ;$ task-irrelevant nonsentence, $\left.F(1,79)=75.68, M S_{\mathrm{e}}=.12, R^{2}=.49, p<.001\right]$.

A 2 (task relevant and task irrelevant) $\times 2$ (sentence and nonsentence) repeated measures analysis of variance (ANOVA) was conducted on the response times for the experimental conditions. The ANOVA indicated a significant main effect of the task relevance of the words. The conditions that included color and color-related words had longer response times $(M=5.416 \mathrm{sec})$ than did the conditions that included only noncolor words $(M=$ $4.697 \mathrm{sec})\left[F(1,79)=200.48, M S_{\mathrm{e}}=.21, R^{2}=.72, p<\right.$ $.001]$. As hypothesized, a significant main effect was also found for sentence structure $\left[F(1,79)=9.71, M S_{\mathrm{e}}=.12\right.$, $\left.R^{2}=.11, p=.003\right]$. Words arranged in sentences $(M=$ $5.117 \mathrm{sec}$ ) caused more interference than did the same words randomly ordered in nonsentences $(M=4.997 \mathrm{sec})$.

In addition, evidence of an interaction between task relevance and sentence structure emerged. Sentences caused greater interference than did nonsentences only when they contained task-relevant words $\left[F(1,79)=13.32, M S_{\mathrm{e}}=\right.$ $\left..11, R^{2}=.14, p<.001\right]$. An individual contrast indicated that the task-relevant sentences were associated with longer response times than were the task-relevant nonsentences $\left[F(1,79)=17.49, M S_{\mathrm{e}}=.15, R^{2}=.18, p<\right.$ $.001]$. For four out of the five stimulus sets, the taskrelevant sentence was associated with a longer response time than was the task-relevant nonsentence. Sentences that included only task-irrelevant words were not associated with longer response times than were nonsentences containing the same words $[F(1,79)<1]$.

\section{Table 1}

Experiment 1 Mean Response Times (With Standard Errors) and Mean Error Proportions (With Standard Errors) by Experimental Condition

\begin{tabular}{|c|c|c|c|c|c|c|c|c|}
\hline \multirow[b]{2}{*}{ Condition } & \multicolumn{4}{|c|}{ Task-Relevant } & \multicolumn{4}{|c|}{ Task-Irrelevant } \\
\hline & RT & $S E$ & EP & $S E$ & $\mathrm{RT}$ & $S E$ & EP & $S E$ \\
\hline & 554 & .101 & .160 & .018 & 4.691 & .074 & .045 & .009 \\
\hline Nonsentence & 5.290 & .107 & .078 & .014 & 4.704 & .079 & .052 & .010 \\
\hline Control & & & & & 4.234 & .074 & .032 & .011 \\
\hline
\end{tabular}

Error rate. Analyses of the error rate data were conducted using both raw proportions and arc sine transformed proportions. The results were nearly identical for these separate analyses. For the sake of brevity, therefore, only the analyses of the untransformed proportions are presented here.

Unlike the response time analyses, individual contrasts of the error proportions indicated that only the taskrelevant conditions differed significantly from the control condition [task-relevant sentence, $F(1,79)=39.83$, $M S_{\mathrm{e}}=.02, p<.001$; task-relevant nonsentence, $F(1,79)=$ 5.93, $M S_{\mathrm{e}}=.01, p=.017$; task-irrelevant sentence, $F(1,79)<1$; task-irrelevant nonsentence, $F(1,79)=1.61$, $\left.M S_{\mathrm{e}}=.01, p=.208\right]$.

The results of a 2 (task relevant and task irrelevant) $\times 2$ (sentence and nonsentence) repeated measures ANOVA of the errors in the experimental conditions mirrored the results of the response time analyses. A significant main effect of the task relevance of the words emerged. Taskrelevant words caused more errors than did task-irrelevant words $(M=.119$ vs. $M=.049)\left[F(1,79)=30.72, M S_{\mathrm{e}}=\right.$ $\left..01, R^{2}=.28, p<.001\right]$. Again, there was evidence for a significant main effect of sentence structure. Words in sentences resulted in more errors than did the same words randomly ordered in nonsentences $(M=.102$ vs. $M=$ $.065)\left[F(1,79)=8.48, M S_{\mathrm{e}}=.01, R^{2}=.10, p=.005\right]$.

Finally, there was evidence for an interaction between the task relevance of the words and sentence structure. Sentence structure resulted in interference in the colornaming task only when task-relevant words were incorporated $\left[F(1,79)=11.87, M S_{\mathrm{e}}=.01, R^{2}=.13, p<.001\right]$. Individual contrasts indicated that task-relevant sentences were associated with more errors than were task-relevant nonsentences $\left[F(1,79)=13.90, M S_{\mathrm{e}}=.02, R^{2}=.15\right.$, $p<.001]$, but that task-irrelevant sentences were not associated with more errors than were task-irrelevant nonsentences $[F(1,79)<1]$. Sentences that included taskrelevant words were associated with more errors than were their nonsentence counterparts for four of the five stimulus sets.

\section{Discussion}

Experiment 1 provided strong evidence that word processing is obligatory. Each of the four experimental conditions was associated with significantly longer response times than was the control condition. However, only the conditions that included highly task-relevant words were significantly different in error rate from the control condition. The overall picture that emerges suggests that the words are processed by participants despite specific instructions to ignore them.

Further, this study corroborates past research indicating that a word's degree of relevance to the color-naming task determines the extent of performance interference that it causes. Stimuli containing highly task-relevant words (i.e., color and color-related words) were associated with significantly longer response times and signif- 
icantly greater error rates than were stimuli containing only task-irrelevant words.

Finally, strong evidence emerged indicating that sentences cause greater interference in the Stroop task than do the same words arranged randomly. Response times were significantly longer and error rates were significantly larger for the sentence conditions than for the nonsentence conditions. These findings are noteworthy, given that response time and error rate differences are not always found concurrently (see, e.g., Levy, Newell, Snyder, \& Timmins, 1986).

The results of Experiment 1 indicate that sentences can be processed without the intention of the reader. Not only were participants unable to ignore the individual words, they were unable to prevent themselves from processing the sentences. Sentence processing in Experiment 1 was demonstrated only for the sentences that included task-relevant words. It is possible, however, that sentence processing occurred in both sentence conditions, but that the nature of the task made it impossible for processing of task-irrelevant sentences to be demonstrated. The traditional Stroop task primes color-relevant words, facilitating their processing, and thus the likelihood that they will compete with the correct ink color names at the output stage. Because the interference inherent to the Stroop task occurs at the response stage, interference may only be evident when stimuli include words that are appropriate responses in the task. When participants in a Stroop-like task were required to name the spatial directions in which words occurred in stimulus squares, interference was greater when the squares included direction words (i.e., left, right, up, or down) than when they included words less relevant to spatial direction (Fox et al., 1971). The requirements of the task made the processing of direction words detrimental to performance, just as the processing of color words is detrimental to performance in the traditional Stroop color-naming task. Words less related to spatial direction than those listed earlier also produced interference, but to a lesser degree. These less relevant words were processed, but because they were not appropriate responses in the task, that processing was more difficult to demonstrate.

In the present study, it was possible that the taskirrelevant sentences also were processed without the participants' intention, but that because the component words were not color words, they did not interfere with the colornaming response. Because these task-irrelevant words were not appropriate responses in the color-naming task, their activation would have had little impact on response selection. The Stroop task measures competition in response selection. Therefore, it is possible that we were simply unable to demonstrate processing of task-irrelevant sentences with the use of the Stroop task.

If noncolor words are capable of producing response interference, as previous research has indicated, the taskirrelevant conditions should have followed a pattern similar to that found in the task-relevant conditions. The fact that heightened processing of words embedded in sen- tences could not be demonstrated for the task-irrelevant stimuli may indicate that the sentences that did not include task-relevant words were not processed at the sentence level. That the task-relevant stimuli were associated with significantly longer response times and more errors than the task-irrelevant stimuli indicates that priming in the Stroop task activates color and color-related words, facilitating their processing relative to that of unprimed, noncolor words. This enhanced word activation may make processing at the sentence level more likely.

If indeed only the task-relevant sentences were processed at the sentence level, this result would have implications for our understanding of what aspects of the sentence context contribute to sentence interference in the Stroop task. Although it is impossible to determine whether the meaning of the sentences or just their syntactic structure interfered with participants' ability to perform the color-naming task, the fact that sentence processing was demonstrated only when the sentences included words highly relevant semantically to the task suggests that participants may indeed have been processing the full meaning of the sentences, rather than just the syntactic structure.

Although unlikely, there are three alternative explanations for the interaction between sentence structure and task relevance in Experiment 1. First, the words in each nonsentence were ordered according to a unique random pattern. The interaction in Experiment 1 might have resulted from differing levels of incoherence in the nonsentences. Perhaps the task-relevant nonsentences were better scrambled and thus more fully devoid of sentence structure and meaning than the task-irrelevant nonsentences. Such a difference, if indeed it existed, might have been the reason that the sentence effect appeared in the task-relevant condition, but not in the task-irrelevant condition.

Second, although the experimental stimuli in Experiment 1 were designed to control for word meaning and word frequency, there was no attempt to control for word length across task relevance conditions. Previous research indicates that short words are more quickly identified in a single eye fixation than long words (e.g., Just $\&$ Carpenter, 1980). Therefore, short words should be more difficult to ignore, and thus more disruptive to Stroop performance, than long words. In Experiment 1, the average number of syllables per stimulus was smaller in the task-relevant condition than in the task-irrelevant condition (12.4 and 17.8, respectively). Therefore, the words in the task-relevant stimuli may have been more difficult to ignore than those in the task-irrelevant stimuli, not only because of their greater relevance to the color-naming task, but also because of their shorter length. Note, however, that word length was identical in the sentence and nonsentence conditions because the same words were used in each case. Therefore, the observed sentence effects cannot be explained in terms of differences in word length.

Third, it is possible that the task-relevant and taskirrelevant stimuli differed with respect to their complexity. Because the task-relevant stimuli included so many 
color and color-related words, which were replaced with noncolor words in the task-irrelevant stimuli, the taskrelevant and task-irrelevant stimuli differed substantially. On the average, only 3.4 words remained constant across these two conditions. Therefore, it is conceivable that the stimuli in these two conditions were not equally complex. If the task-relevant sentences were less complex on the average than the task-irrelevant sentences, the former may have been easier to process, thus causing greater interference in the color-naming task and potentially a larger difference between the sentence and nonsentence conditions.

To obtain a clearer understanding of the nature of the interaction between task relevance and sentence structure, a further experiment was conducted in which word randomization, word length, degree of task relevance, and stimulus complexity were strictly controlled.

\section{EXPERIMENT 2}

Experiment 1 provided strong evidence for sentence activation under conditions of high task relevance. This experiment, however, did not fully utilize the strongest manipulation possible in the Stroop task. Klein (1964) demonstrated that the "standard" Stroop condition, in which incongruently printed color words correspond to or match the set of ink colors in which the stimuli are printed, causes greater interference than do conditions in which the color words represent colors not used in printing the stimuli. For example, the word red printed in blue ink is associated with longer response times when red is one of the ink colors used to print the stimuli than when it is not. Output interference is enhanced by the fact that the color word to be suppressed is a valid response in the color-naming task.

In Experiment 1, no attempt was made to match the set of color words to the set of ink colors in which the stimuli were printed. Nonetheless, every task-relevant stimulus included at least one, but usually only one, color word corresponding to one of the ink colors used to print the stimuli (i.e., red, blue, or green). On the average, 1.2 words in a given task-relevant stimulus matched the set of ink colors. Given that such matching words have the greatest relevance to the color-naming task, it would seem that the stimuli in Experiment 1 might not have provided the strongest possible demonstration of obligatory sentence activation in the Stroop task.

In Experiment 2, as an additional test of obligatory sentence processing, sentence interference was maximized by utilizing multiple matching color words per stimulus. It was hypothesized that an effect of sentence structure would be most evident when the component set of incongruently printed color words matched the set of ink colors used to print the stimuli (highest relevance). Identical sentences printed in a different, and mismatching, set of ink colors were also expected to show evidence of sentence processing (lower relevance). It was predicted, however, that these less relevant mismatching stimuli would show less interference at the level of the sentence than would sentences containing highly relevant matching color words. Because the sentence and nonsentence stimuli in Experiment 2 were identical across the highest relevance and lower relevance conditions in every respect except for the ink colors in which they were printed, this study provided an excellent test of the effects of task relevance, sentence structure, and their interaction while completely eliminating any possible impact of word length and stimulus complexity. Further, the use of the same word randomization across nonsentence conditions ensured that sentence structure and task relevance were not confounded.

As in Experiment 1, it was hypothesized that words arranged in meaningful sentences would cause more interference than would the same words arranged randomly. Further, it was expected that stimuli that contained words of the highest possible task relevance (i.e., color words that were in the ink color response set) would cause more interference than would stimuli containing words of lower task relevance (i.e., color words that were not included in the response set). In addition, an interaction of sentence structure and task relevance was expected because interference should be greatest for sentences containing color words that match the ink color response set.

\section{Method}

Participants. The participants were 114 University of Colorado undergraduate students. Although 127 students participated in the experiment, 13 were replaced for reasons described in the Results section. Thirty of the participants were paid for their participation, whereas the other 84 participated in partial fulfillment of a course requirement. All participants had normal color vision.

Design, Materials, and Apparatus. In Experiment 2, both of the task relevance conditions included task-relevant words. Task relevance was manipulated by generating stimuli that included words that were of the highest relevance to the color-naming task (i.e., color words that matched the set of ink colors used to print the stimuli) and stimuli that were comparatively less relevant to the color-naming task (i.e., color words that did not match the ink colors). The experimental design consisted of six within-subjects conditions: (1) sentences in which the set of color words, although always printed incongruently, matched the set of ink colors used to print the stimuli (highest relevance sentence condition), (2) sentences in which the set of incongruent color words did not match the set of ink colors (lower relevance sentence condition), (3) nonsentences in which the set of incongruent color words matched the set of ink colors (highest relevance nonsentence condition), (4) nonsentences in which the set of incongruent color words did not match the set of ink colors (lower relevance nonsentence condition), (5) control stimuli consisting of groupings of bullets printed in color set $A$, which consisted of the ink colors red, orange, and green (control A), and (6) control stimuli consisting of groupings of bullets printed in color set $B$, which consisted of the ink colors purple, blue, and brown (control B).

Six stimulus sets were generated. Each set included six stimuli, one stimulus corresponding to each of the six conditions. All of the sentences contained nine words, three of which were color words. For three sets, the color words were red, orange, and green (color word set A), and for the other three sets, the color words were purple, blue, and brown (color word set B). The sentences were printed in either ink color set $\mathrm{A}$ (red, orange, and green) or ink color set $\mathrm{B}$ (purple, blue, and brown). For sentences in the highest relevance condition, the set of color words corresponded to the set of ink col- 
ors used in printing the stimuli (e.g., a sentence including the three words from color word set A printed incongruently in the three colors from ink color set A). For sentences in the lower relevance condition, the set of color words did not correspond to the set of ink colors (e.g., a stimulus including the three words from color word set A printed incongruently in the three ink colors from ink color set $\mathrm{B}$ ).

In each stimulus set, the two sentences were identical, except that one was printed in ink color set $A$, whereas the other was printed in ink color set $\mathrm{B}$. Because the highest relevance and lower relevance stimuli within each set included the same words, the numbers of syllables in these stimuli were necessarily identical. The nonsentences were generated by randomizing the word order of the sentences. The two nonsentences in each stimulus set were identically scrambled versions of the sentences. As with the sentences, the nonsentences differed only in that one was printed in ink color set $A$, and the other in ink color set B. Therefore, word length, sentence complexity, and word randomization, which might have contributed to the interaction between task relevance and sentence structure in Experiment 1, were eliminated as possible sources of variance in Experiment 2. Appendix B contains the sentences from each of the stimulus sets, as well as the mean response times and error frequencies by condition for each set.

To control for possible differences in the clarity or identifiability of the ink colors in the two ink color sets, two control stimuli were generated for each stimulus set. These two stimuli were identical to each other, with the exception that one was printed in ink color set A, whereas the other was printed in ink color set $\mathrm{B}$. The control stimuli were modeled after the sentence stimuli. Each control was composed of nine groupings of bullets, corresponding to the nine words in each sentence stimulus. Each of the nine groupings of bullets included as many individual bullets as the words in the same positions in the sentences contained letters.

Unlike in Experiment 1, the 36 stimuli in Experiment 2 were printed in lowercase letters, with the exception of the first letter of the first word in each sentence and nonsentence, which was capitalized. Lowercase letters were used in Experiment 2 to increase the readability of the words. Each sentence, nonsentence, and control stimulus was printed horizontally across its own card. Each stimulus was printed in either ink color set A or ink color set B. Ink colors were assigned to particular words within a stimulus according to the same four rules that had been used in Experiment 1. First, each of the three colors in an ink color set was used three times in each stimulus. Second, no color was used twice in succession. Third, ink color and word combinations were always incongruent. Finally, to avoid the distractor-suppression effect, the ink color of a given word in a stimulus never matched the preceding color word. Again, each stimulus within a stimulus set was characterized by a unique sequence of ink colors. As in Experiment 1, the stimuli were ordered according to a Greco-Latin square design. Six presentation orders were used. Nineteen participants were tested with each order. Each participant was exposed to each of the 36 stimuli.

Procedure. The participants received written and oral instructions. Prior to participating in the Stroop task, the participants were shown sets of colored Xs to familiarize them with the six ink colors. The participants then engaged in the Stroop task on six sample items. These sample stimuli were presented on individual $8.5 \times$ $11 \mathrm{in} .(21.6 \times 27.9 \mathrm{~cm})$ sheets of paper. Each sample stimulus was composed of nine sets of $\mathrm{Xs}$ of varying lengths and was printed in one of the two ink color sets. The sample items provided a test of the participants' color vision. No participants had to be eliminated on the basis of color-blindness.

Errors and response times were collected by a naive experimenter unaware of the experimental hypotheses, using the same procedures as in Experiment 1. As in Experiment 1, the experimental participants controlled the stimulus cards. The experimenter could not see the stimuli on the cards and thus was unaware of the condition to which participants were responding at any given time.

\section{Results}

As in Experiment 1, all stimuli on which errors were committed were removed from the analysis of the response times for that participant. Further, when an error was committed on a given stimulus, the entire stimulus set to which that item belonged was removed from the analysis of response times for that participant. Out of a total of 4,104 stimuli, 537 were eliminated from the response time analysis because of errors. For 461 of these errors, participants incorrectly named the ink colors. Thirty items were dropped because of participants' errors in following directions, and 37 items were dropped because of experimenter timing errors. Nine response times fell more than three standard deviations away from the mean for the corresponding stimuli and were removed from the analysis. Thirteen participants were dropped from the data set entirely because of errors or outlying values in every stimulus set. All participants who were removed from the analyses were replaced with participants who had been tested with the same stimulus order as that for the individuals who had been dropped.

Response time. The response time data were analyzed using both the raw data and the data transformed to the natural $\log$ with base $e$. These separate analyses resulted in identical conclusions. Only the analysis of the raw data, therefore, is reported. The condition means for the response time and error rate data, along with their standard errors, are presented in Table 2. To determine whether the experimental conditions differed from the controls, each experimental stimulus was compared with the control stimulus in its stimulus set that was printed in the same color set. Individual contrasts indicated that each of the four experimental conditions was associated with significantly longer response times than were the control conditions [highest relevance sentence, $F(1,113)=277.87$, $M S_{\mathrm{e}}=.35, p<.001$; lower relevance sentence, $F(1,113)=$ $110.22, M S_{\mathrm{e}}=.31, p<.001$; highest relevance nonsentence, $F(1,113)=178.95, M S_{\mathrm{e}}=.36, p<.001$; lower relevance nonsentence, $F(1,113)=107.45, M S_{\mathrm{e}}=.37$, $p<.001]$.

A 2 (highest relevance and lower relevance) $\times 2$ (sentence and nonsentence) repeated measures ANOVA of the response times indicated a powerful effect of the task relevance manipulation $\left[F(1,113)=13.43, M S_{\mathrm{e}}=.51\right.$, $\left.R^{2}=.11, p<.001\right]$. As predicted, the highest relevance stimuli $(M=6.042 \mathrm{sec})$ were associated with longer re-

Table 2

Experiment 2 Mean Response Times (With Standard Errors) and Mean Error Proportions (With Standard Errors) by Experimental Condition

\begin{tabular}{|c|c|c|c|c|c|c|c|c|}
\hline \multirow[b]{2}{*}{ Condition } & \multicolumn{4}{|c|}{ Highest Relevance } & \multicolumn{4}{|c|}{ Lower Relevance } \\
\hline & RT & $S E$ & EP & $S E$ & RT & $S E$ & EP & $S E$ \\
\hline Sentence & 6.164 & .082 & .190 & .015 & 5.766 & .087 & .107 & .012 \\
\hline $\begin{array}{l}\text { Nonsentence } \\
\text { Controls }\end{array}$ & 5.920 & .091 & .160 & .014 & 5.829 & .090 & .075 & .012 \\
\hline Color set A & & & & & 4.790 & .070 & .065 & .010 \\
\hline Color set B & & & & & 5.062 & .069 & .089 & .011 \\
\hline
\end{tabular}


sponse times than were the lower relevance stimuli $(M=$ $5.798 \mathrm{sec}$ ). No significant main effect of sentence structure emerged in this overall analysis $[F(1,113)=2.47$, $\left.M S_{\mathrm{e}}=.38, R^{2}=.02, p=.119\right]$. It should be noted that, whereas the mean response time of the highest relevance sentence condition $(M=6.164 \mathrm{sec})$ was longer than that of the highest relevance nonsentence condition $(M=$ $5.920 \mathrm{sec})$, as predicted, the lower relevance sentences $(M=5.766 \mathrm{sec})$ were actually associated with a slightly shorter mean response time than were the lower relevance nonsentences $(M=5.829 \mathrm{sec})$. This reversal of direction contributed to a significant interaction between sentence structure and task relevance condition $[F(1,113)$ $\left.=8.14, M S_{\mathrm{e}}=.33, R^{2}=.08, p=.005\right]$.

To clarify the impact of sentence structure on response times, two ANOVAs were conducted to compare the sentence with the nonsentence conditions separately for the highest relevance and lower relevance conditions. For the highest relevance condition, sentences resulted in longer response times than did nonsentences $[F(1,113)=8.66$, $\left.M S_{\mathrm{e}}=.39, R^{2}=.08, p=.004\right]$. This relationship was found for three of the six stimulus sets. No effect of sentence structure appeared in the lower relevance condition $[F(1,113)<1]$.

Error Rate. Individual contrasts indicated that only the highest relevance conditions were associated with significantly larger error proportions than were the control conditions [highest relevance sentence, $F(1,113)=$ 53.66, $M S_{\mathrm{e}}=.02, p<.001$; lower relevance sentence, $F(1,113)=1.62, M S_{\mathrm{e}}=.01, p=.206$; highest relevance nonsentence, $F(1,113)=35.72, M S_{\mathrm{e}}=.01, p<.001$; lower relevance nonsentence, $F(1,113)<1]$.

A 2 (highest relevance and lower relevance) $\times 2$ (sentence and nonsentence) repeated measures ANOVA of the error data indicated a significant effect of task relevance condition $\left[F(1,113)=53.66, M S_{\mathrm{e}}=.02, R^{2}=.30\right.$, $p<.001]$. Stimuli in which the set of color words matched the ink color set in which the stimuli were printed were associated with more errors $(M=.174)$ than were stimuli in which the color words did not match the ink colors making up the color set $(M=.091)$. In addition, a significant main effect of sentence structure emerged $[F(1,113)=$ $\left.5.99, M S_{\mathrm{e}}=.02, R^{2}=.05, p=.016\right]$. Participants committed more errors when performing the Stroop task with sentences $(M=.148)$ than with nonsentences $(M=$ .118). More errors were committed on highest relevance sentences than on highest relevance nonsentences for five of the six stimulus sets. Further, more participants committed errors on lower relevance sentences than on the corresponding lower relevance nonsentences for four out of the six stimulus sets. No interaction between sentence structure and task relevance emerged $[F(1,113)<1]$.

\section{Discussion}

Experiment 2 corroborated past evidence indicating that stimuli including incongruent color words that match the set of ink colors used to print the stimuli cause greater interference in the Stroop task than do stimuli in which the incongruent color words do not match the set of ink colors. Highest relevance stimuli were associated with both longer response times and more errors than were lower relevance stimuli.

Experiment 2 contributed further evidence that sentences can be processed without the intention of the reader. Participants committed more errors overall on sentence stimuli than on nonsentence stimuli. Further, when the component color words matched the set of ink colors used to print the stimuli, sentences were associated with longer response times than were nonsentences. As in Experiment 1 , the strongest evidence for sentence processing emerged when the component words were especially relevant to the task at hand.

We had expected to find evidence of sentence processing in both the highest relevance and the lower relevance conditions of Experiment 2. That such processing was not evident for response times in the lower relevance sentences may appear to be at odds with the results of Experiment 1 . After all, the lower relevance condition included color words, as did the task-relevant condition in Experiment 1, which showed evidence of sentence activation. However, all of the task-relevant stimuli in the previous experiment included one or two words of the highest possible task relevance (i.e., color words that matched the set of ink colors). The two experiments, therefore, yield consistent evidence that sentence processing is obligatory when stimuli include at least one word that matches the set of ink colors used to print the stimuli.

The interaction between task relevance condition and sentence structure in the response time analyses occurred despite the fact that the sentences were identical and the nonsentences were identical across task relevance conditions. This interaction leads to greater confidence that the similar interaction in Experiment 1 was not simply a result of differences in word randomization, sentence complexity, or word length across task relevance conditions.

One possible alternative explanation for the results of Experiment 2 involves the ordering of the color words across the sentence and nonsentence conditions. It is possible that differences in Stroop interference resulted from differences in the positions of the color words across sentence and nonsentence conditions. Although differing positions of the color words might have artificially increased or decreased the impact of sentence context, it cannot explain why the sentence effect differed in the highest relevance and lower relevance conditions. The order of the color words was identical in the two sets of sentences. Likewise, the order was identical in the two sets of nonsentences. Therefore, if the sentence effects are the result of color word position, those effects should have been parallel in the highest and lower relevance conditions. However, that was not the case. The sentence effect on response time was apparent only for stimuli that included color words matching the set of ink colors used to print the stimuli. 


\section{GENERAL DISCUSSION}

The experiments reported here were designed to determine whether or not obligatory processing in reading occurs at the sentence level as well as the word level. Our analyses replicated findings of obligatory processing at the word level. With the exception of the task-irrelevant and lower relevance conditions in the error rate analyses, all of the experimental conditions were associated with longer response times and more errors than were the corresponding control conditions. Most of the time, the words received processing despite specific instructions to ignore them and despite the detrimental effect that processing the words had on performance in the colornaming task.

These experiments also indicated a powerful effect of the task relevance of the stimuli. In Experiment 1, stimuli that included color and color-related words were associated with longer response times and more errors than were stimuli that included only task-irrelevant words. In Experiment 2, stimuli including color words that matched the set of ink colors in which the stimuli were printed were associated with longer response times and more errors than were the stimuli in which the set of color words did not match the set of ink colors.

This study also provided evidence that sentences can be processed without the intention of the reader. In Experiment 1 , sentences were associated with longer response times and more errors than were nonsentences. However, this relationship was found only when the stimuli included color and color-related words. In Experiment 2 , sentences were associated with more errors than were nonsentences overall, but they were associated with longer response times than were nonsentences only when they contained color words that matched the set of ink colors used to print the stimuli. Across the two experiments, sentence interference was demonstrated in the conditions in which the stimuli included color words that matched the set of ink colors. Sentence processing was not demonstrated consistently in the other conditions.

These experiments suggest that sentence processing can occur without the intention of the reader when the sentences' component words are highly relevant to the task. However, these experiments cannot eliminate the possibility that the sentences were activated in all of the task relevance conditions. We may have been able to demonstrate sentence processing most clearly in the high task relevance conditions because some of the words included in those stimuli corresponded to the responses in the color-naming task. The component words in the less task-relevant conditions were less likely to compete with the appropriate color-naming responses, however, thus producing less interference. Therefore, the less taskrelevant sentences may have been processed, but without producing interference in the Stroop task.

Although the present findings suggest that sentences can be processed without the intention of the reader, it is unclear whether participants processed sentence meaning or sentence structure. Because of the difficulty of generating meaningful sentences that include multiple color words as well as corresponding sentences that do not but are matched on a number of dimensions, the sentences used in Experiments 1 and 2 were somewhat contrived. This aspect of the stimuli may suggest that the experimental participants were primarily processing the syntax of these sentences, rather than the meaning. However, the fact that the sentences were fairly contrived makes the consistent effect of sentence context even more noteworthy.

Further, there is reason to believe that participants were processing the meaning of the sentences. Simpson et al. (1989) demonstrated that sentence effects in a word-naming task were not simply a result of activation of the sentence structure, but of the sentence meaning. In the present study, the fact that sentences were processed primarily when they included task-relevant words suggests that it was the meaning and not simply the structure of the sentences that received processing. If only sentence structure was processed, all sentences should have caused equal interference in the color-naming task, regardless of the task relevance of their component words. In Experiment 2, in particular, the only way in which the sentence stimuli differed across task relevance conditions was in the semantic relationship between the component color words and the ink colors. This result points strongly to the activation of sentence meaning rather than simply sentence structure. The present study, however, cannot eliminate the possibility that participants responded to sentence structure alone. Further research with well-formed but meaningless sentences could help resolve this remaining issue.

Likewise, although this work has provided evidence for the obligatory processing of whole sentences, the possibility exists that the sentence effects observed may not have occurred at the sentence level at all, but at some level lower than the sentence, yet higher than the word. For example, processing may have occurred at the phrase level rather than at the level of full sentences. It is interesting to note, however, that, using a subliminal priming paradigm, Draine (1997) found no evidence for semantic processing of two-word phrases, although he did find evidence for semantic processing of the constituent words. Further research in this area should be designed to determine precisely the basic unit of obligatory processing when multiple words are involved.

\section{REFERENCES}

Bakan, P., \& Alperson, B. (1967). Pronounceability, attensity, and interference in the color-word test. American Journal of Psychology, 80, 416-420.

Cohen, J. D., Dunbar, K., \& McClelland, J. L. (1990). On the control of automatic processes: A parallel distributed processing account of the Stroop effect. Psychological Review, 97, 332-361.

DALRYMPle-AlFord, E. C. (1972). Associative facilitation and interference in the Stroop color-word task. Perception \& Psychophysics, 11, 274-276.

Dalrymple-Alford, E. C., \& Budayr, B. (1966). Examination of 
some aspects of the Stroop color-word test. Perceptual \& Motor Skills, 23, 1211-1214.

DeSchepPer, B., \& Treisman, A. (1996). Visual memory for novel shapes: Implicit coding without attention. Journal of Experimental $P$ sychology: Learning, Memory, \& Cognition, 22, 27-47.

DRAINE, S. C. (1997). Analytic limitations of unconscious language processing. Unpublished doctoral dissertation, University of Washington.

Fox, L. A., Shor, R. E., \& Steinman, R. J. (1971). Semantic gradients and interference in naming color, spatial direction, and numerosity. Journal of Experimental Psychology, 91, 59-65.

Hess, D. J., Foss, D. J., \& Carroll, P. (1995). Effects of global and local context on lexical processing during language comprehension. Journal of Experimental Psychology: General, 124, 62-82.

Juid, C. M., \& McClelland, G. H. (1989). Data analysis: A modelcomparison approach. San Diego: Harcourt Brace Jovanovich.

JUST, M. A., \& CARPENTER, P. A. (1980). A theory of reading: From eye fixations to comprehension. Psychological Review, 87, 329-354.

KLEIN, G. S. (1964). Semantic power measured through the interference of words with color-naming. American Journal of Psychology, 77, 576-588.

KuČERA, H., \& FranCIS, W. N. (1967). Computational analysis of presentday American English. Providence, RI: Brown University Press.

Levy, B. A., Newell, S., Snyder, J., \& Timmins, K. (1986). Processing changes across reading encounters. Journal of Experimental Psychology: Learning, Memory, \& Cognition, 12, 467-478.

MACLEOD, C. M. (1991). Half a century of research on the Stroop effect: An integrative review. Psychological Bulletin, 109, 163-203.

Masson, M. E. J. (1986). Comprehension of rapidly presented sentences: The mind is quicker than the eye. Journal of Memory \& Language, 25, 588-604.

NEILL, W. T. (1977). Inhibitory and facilitatory processes in selective attention. Journal of Experimental Psychology: Human Perception \& Performance, 3, 444-450.
NeiLl, W. T., \& Westberry, R. L. (1987). Selective attention and the suppression of cognitive noise. Journal of Experimental Psychology: Learning, Memory, \& Cognition, 13, 327-334.

O'SeaghdHa, P. G. (1989). The dependence of lexical relatedness effects on syntactic connectedness. Journal of Experimental Psychology: Learning, Memory, \& Cognition, 15, 73-87.

Posner, M. I., \& SNyder, C. R. R. (1975). Attention and cognitive control. In R. L. Solso (Ed.), Information processing and cognition: The Loyola symposium (pp. 55-85). Hillsdale, NJ: Erlbaum.

RUMELHART, D. E. (1977). Toward an interactive model of reading. In S. Dornic (Ed.), Attention and performance VI (pp. 573-603). Hillsdale, NJ: Erlbaum.

Samar, V. J., \& Berent, G. P. (1986). The syntactic priming effect: Evoked response evidence for a prelexical locus. Brain \& Language, 28, 250-272.

SERENO, J. A. (1991). Graphemic, associative, and syntactic priming effects at a brief stimulus onset asynchrony in lexical decision and naming. Journal of Experimental Psychology: Learning, Memory, \& Cognition, 17, 459-477.

ShADLER, M., \& ThisSEN, D. M. (1981). The development of automatic word recognition and reading skill. Memory \& Cognition, 9, 132-141.

Simpson, G. B., Peterson, R. R., Casteel, M. A., \& Burgess, C. (1989). Lexical and sentence context effects in word recognition. Journal of Experimental Psychology: Learning, Memory, \& Cognition, $15,88-97$.

STROOP, J. R. (1935). Studies of interference in serial verbal reactions. Journal of Experimental Psychology, 18, 643-662.

Tanenhaus, M. K., \& Trueswell, J. C. (1995). Sentence comprehension. In J. L. Miller \& P. D. Eimas (Eds.), Speech, language, and communication (pp. 217-262). San Diego: Academic Press.

WIKE, E. L., \& ChurCh, J. D. (1976). Comments on Clark's "Thelanguage-as-fixed-effect fallacy." Journal of Verbal Learning \& Verbal Behavior, 15, 249-255.

\section{APPENDIX A \\ Sentences Used in Experiment 1}

Underscored words are unique to the task-relevant or the task-irrelevant sentence.

All other words are repeated in both sentences.

Stimulus Set 1

Task-relevant: THE CHARCOAL COLORED SMOKE BLACKENED THE INDIGO BLUE SKY

Task-irrelevant: THE EMPTY MOVING BOXES CLUTTERED THE REMARKABLY ATTRACTIVE KITCHEN

Stimulus Set 2

Task-relevant: BLOOD RED LAVA FLOWED OVER THE BLACK SAND BEACH

Task-irrelevant: RISING FLOOD WATER FLOWED OVER THE FAILING EMERGENCY EMBANKMENT

Stimulus Set 3

Task-relevant: THE SUNSET MADE THE BLUE SKY RED AND PURPLE

Task-irrelevant: THE PROFESSOR MADE THE COMPLICATED THEORY CLEAR AND CONCISE

Stimulus Set 4:

Task-relevant: RED-TAILED SILVER JETS FLEW ACROSS THE FIERY ORANGE SKY

Task-irrelevant: FAST-MOVING FIGHTER JETS FLEW ACROSS THE HASTILY ABANDONED HEADQUARTERS

Stimulus Set 5:

Task-relevant: FIRE TURNED THE FOREST INTO RED AND YELLOW FLAMES

Task-irrelevant: PIONEERS TURNED THE MOUNTAINSIDES INTO SMALL AND THRIVING 
Table A1

Mean Word Frequency by Stimulus Set

\begin{tabular}{|c|c|c|c|c|c|}
\hline \multirow[b]{2}{*}{$\begin{array}{l}\text { Stimulus } \\
\text { Set }\end{array}$} & \multicolumn{3}{|c|}{ Color Stimuli } & \multicolumn{2}{|c|}{ Mean Word Frequency } \\
\hline & $\begin{array}{l}\text { Frequency of } \\
\text { Color Words }\end{array}$ & $\begin{array}{c}\text { Frequency } \\
\text { Color-Related Words }\end{array}$ & $\begin{array}{c}\text { Total } \\
\text { Frequency }\end{array}$ & $\begin{array}{c}\text { Task-Relevant } \\
\text { Stimuli } \\
\end{array}$ & $\begin{array}{c}\text { Task-Irrelevant } \\
\text { Stimuli } \\
\end{array}$ \\
\hline 1 & $3(33 \%)$ & $4(44 \%)$ & $7(78 \%)$ & 15,582 & 15,587 \\
\hline 2 & $2(22 \%)$ & $4(44 \%)$ & $6(67 \%)$ & 7,980 & 7,977 \\
\hline 3 & $3(33 \%)$ & $2(22 \%)$ & $5(56 \%)$ & 18,927 & 18,928 \\
\hline 4 & $3(33 \%)$ & $2(22 \%)$ & $5(56 \%)$ & 7,822 & 7,822 \\
\hline 5 & $2(22 \%)$ & $3(33 \%)$ & $5(56 \%)$ & 11,272 & 11,277 \\
\hline
\end{tabular}

Table A2

Mean Response Times and Error Frequencies by Stimulus Set and Condition

\begin{tabular}{|c|c|c|c|c|c|c|c|c|c|c|}
\hline \multirow{2}{*}{$\begin{array}{c}\text { Stimulus } \\
\text { Set }\end{array}$} & \multicolumn{2}{|c|}{$\begin{array}{l}\text { Task-Relevant } \\
\text { Sentence } \\
\end{array}$} & \multicolumn{2}{|c|}{$\begin{array}{c}\text { Task-Irrelevant } \\
\text { Sentence }\end{array}$} & \multicolumn{2}{|c|}{$\begin{array}{l}\text { Task-Relevant } \\
\text { Nonsentence } \\
\end{array}$} & \multicolumn{2}{|c|}{$\begin{array}{c}\text { Task-Irrelevant } \\
\text { Nonsentence }\end{array}$} & \multicolumn{2}{|c|}{ Control } \\
\hline & RT & $\mathrm{EF}$ & RT & EF & RT & $E F$ & RT & $\mathrm{EF}$ & RT & $\mathrm{EF}$ \\
\hline 1 & 5.286 & 21 & 4.606 & 0 & 5.255 & 6 & 4.525 & 3 & 4.197 & 2 \\
\hline 2 & 5.311 & 19 & 4.334 & 1 & 4.836 & 1 & 4.365 & 2 & 4.025 & 2 \\
\hline 3 & 5.427 & 18 & 4.681 & 10 & 5.101 & 21 & 4.417 & 2 & 4.107 & 6 \\
\hline 4 & 4.918 & 2 & 4.692 & 7 & 4.921 & 1 & 4.755 & 14 & 4.055 & 4 \\
\hline 5 & 5.522 & 8 & 4.521 & 2 & 5.277 & 6 & 4.771 & 6 & 4.185 & 3 \\
\hline
\end{tabular}

\section{APPENDIX B \\ Sentences Used in Experiment 2}

Note-Color words are in italics.

Stimulus Set 1: The red and orange boxes cluttered the green kitchen

Stimulus Set 2: Orange and red flowers bloomed in the green pot

Stimulus Set 3: Red jets flew across the green and orange pavement

Stimulus Set 4: Purple and blue labels covered the brown glass bottles

Stimulus Set 5: Blue and purple flags flew atop the brown building

Stimulus Set 6: Blue and purple parachutes dropped from the brown airplane

Table B1

Mean Response Times and Error Frequencies by Stimulus Set and Condition

\begin{tabular}{|c|c|c|c|c|c|c|c|c|c|c|c|c|}
\hline \multirow{2}{*}{$\begin{array}{l}\text { Stimulus } \\
\text { Set }\end{array}$} & \multicolumn{2}{|c|}{$\begin{array}{c}\text { Highest Relevance } \\
\text { Sentence }\end{array}$} & \multicolumn{2}{|c|}{$\begin{array}{c}\text { Lower Relevance } \\
\text { Sentence } \\
\end{array}$} & \multicolumn{2}{|c|}{$\begin{array}{l}\text { Highest Relevance } \\
\text { Nonsentence }\end{array}$} & \multicolumn{2}{|c|}{$\begin{array}{c}\text { Lower Relevance } \\
\text { Nonsentence }\end{array}$} & \multicolumn{2}{|c|}{ Control A } & \multicolumn{2}{|c|}{ Control B } \\
\hline & $\mathrm{RT}$ & $\mathrm{EF}$ & RT & $E F$ & RT & EF & RT & $\mathrm{EF}$ & RT & $\mathrm{EF}$ & RT & EF \\
\hline 1 & 6.026 & 15 & 6.240 & 24 & 6.089 & 12 & 6.082 & 5 & 4.955 & 4 & 5.143 & $\overline{9}$ \\
\hline 2 & 6.413 & 20 & 6.066 & 16 & 5.606 & 13 & 6.197 & 11 & 4.836 & 8 & 5.283 & 15 \\
\hline 3 & 5.886 & 12 & 5.607 & 5 & 5.950 & 9 & 5.792 & 11 & 4.622 & 6 & 5.076 & 9 \\
\hline 4 & 6.356 & 25 & 5.697 & 2 & 5.683 & 20 & 5.695 & 14 & 4.971 & 11 & 5.072 & 3 \\
\hline 5 & 6.054 & 17 & 5.478 & 10 & 6.148 & 15 & 5.555 & 4 & 4.779 & 3 & 4.878 & 5 \\
\hline 6 & 6.249 & 38 & 5.604 & 15 & 5.801 & 39 & 5.553 & 6 & 4.942 & 12 & 5.204 & 18 \\
\hline
\end{tabular}

(Manuscript received November 17, 1997;

revision accepted for publication September 8, 1998.) 